\title{
Stereological estimation of ovarian oocyte volume, surface area and number: application on mice treated with nandrolone decanoate
}

\author{
Saied Karbalay-Doust, Ali Noorafshan \\ Histomorphometry and Stereology Research Center, Shiraz University of Medical Sciences, \\ Shiraz, Iran
}

\begin{abstract}
Changes in the number and size of oocytes can lead to fertilization problems. The present study aimed to evaluate the number, volume, and surface area of oocytes in healthy as well as nandrolone decanoate-treated (ND) mice using stereological methods. Five control mice received vehicle, and five ND-treated mice received ND. Using the 'isotropic Cavalieri' design', the ovary was sectioned. The volume of the ovary (cortex and medulla) was estimated. The oocytes' volume and surface area were estimated using the invariator. The number of the oocytes was estimated using an optical disector. The volumes of the ovary, cortex, and medulla decreased $\sim 50 \%$ in the ND-treated mice. The mean number (coefficient of variation) of preantral, antral, and atretic oocytes in the control ovary were 1,690 (0.29), 2,100 (0.52), and 3,900 (0.2), respectively, which decreased $\sim 54 \%, \sim 87 \%$, and $\sim 91 \%$, respectively in the ND-treated animals. The mean volume (coefficient of variation) of the preantral, antral, and atretic oocytes were 86,000 (0.27), 110,000 (0.48), and 27,000 (0.33) $\mu \mathrm{m}^{3}$, respectively. The mean surface area (coefficient of variation) of the three types of oocytes were 9,000 (0.24), 9,900 (0.28), and 4,700 $(0.21) \mu \mathrm{m}^{2}$, respectively. These parameters remained unchanged in the ND-treated mice. ND induces reduction in the number of oocytes, but not in the volume or the surface area. (Folia Histochemica et Cytobiologica 2012, Vol. 50, No. 2, 275-279)
\end{abstract}

Key words: stereology, ovary, oocyte, nandrolone decanoate, mouse

\section{Introduction}

The ovary and its productions, the oocytes, can be, directly or indirectly, influenced by pathological conditions. Nandrolone decanoate (ND) is a subtype of androgenic anabolic steroid. Such steroids are synthetic analogs of the males' main reproductive hormone, i.e. testosterone, and are prescribed for the treatment of some medical conditions including renal insufficiency, endometriosis, and hereditary angioedema [1]. Some athletes (and non-athletes) abuse steroids in order to increase their muscular mass,

Correspondence address: A. Noorafshan,

Histomorphometry and Stereology Research Center,

Shiraz University of Medical Sciences,

71348-45794, Zand Avenue, Shiraz, Iran;

tel./fax: + 987112304372 ;

e-mail:noora@sums.ac.ir power, and endurance [2]. The side effects of anabolic steroids on the physiology and function of the reproductive system have been proved [3]. The side effects of steroids on the histology of testis [4], prostate [5], ovary, and uterus [6-7], spermatogenesis [8], genitalia malformation, and ovarian cyclical activity [1, 9-11] have also been shown. Ovarian and uterine histology and follicular score have been investigated after the ND treatment [6, 7]; however, quantitative aspects of the ovary and oocytes have received little attention. Stereological methods can provide the quantitative parameters of the ovary and oocytes which might not be detectable using qualitative evaluations. The volume of the ovary and its cortical and medullary regions was estimated. In addition, the number, the volume, and the surface of oocytes in the preantral, antral, and atretic follicles were estimated. Changes in these histological parameters may lead to fertilization. For example, the surface area of 
oocytes is the entrance of the spermatozoon. The present research was conducted in order to quantify the histological parameters of mouse ovaries after ND treatment.

\section{Material and methods}

Animals and tissue preparation. Ten BALB/c female mice were randomly assigned to either a control $(n=5)$ or an ND-treated $(n=5)$ group. The animals were kept under constant conditions of light (12 hours light/dark cycle) and temperature $\left(21-24^{\circ} \mathrm{C}\right)$. All the mice had ad libitum access to food and water. All investigations were conducted in accordance with the ethical principles of the use of laboratory animals adopted by Shiraz University of Medical Sciences. Vehicle (olive oil) and ND were intraperitoneally injected to the control and the experimental group, respectively. ND was injected in doses of $3 \mathrm{mg} / \mathrm{kg}$ of body weight. It was administered in one, two and three $\mathrm{mg} / \mathrm{kg}$ doses in the first, second, and third week of treatment, respectively [4]. The mice were euthanized by inhalation of ethyl ether 72 hours after the last injection. The left ovaries were removed and weighed. Then, the ovaries were fixed in $4 \%$ formaldehyde buffer. Isotropic uniform random sections are necessary in order to estimate some stereological parameters including the oocyte's surface area [12-15]. The ovaries should be sectioned based on the 'isotropic Cavalieri' design to emphasize the fact that the sections are parallel and hence not independent and can be used in estimation of the ovary volume $[13,15]$. To obtain these sections, a method called 'isector' was applied. Briefly, each ovary was embedded in a spherical module filled with paraffin, randomly rotated, and serially sectioned (sections of $15 \mu \mathrm{m}$ thickness) [16]. Eight to twelve sections from each ovary were sampled through systematic random sampling and were stained by Heidenhain's Azan trichrome.

Estimation of the volume of the ovary, cortex, and medulla. Estimation of the total ovarian volume, 'V(Ref)', was made according to the Cavalieri principle and using a stereomicroscope connected to a computer, at the final magnification of $20 \times$. A brief description is presented under Figure $1[13,15]$.

Identification of the oocytes. All the oocytes were distinguishable from somatic cells by a characteristically spherical nucleus, a considerably larger nucleus, and the cell size. The following classification was used for recognition of each oocyte subpopulation during the stereological counting:

- preantral oocyte was distinguished by its distinct cytoplasm and prominent nucleus which was surrounded by single or several layers of follicular cells without any space (antrum) between the cells;

- antral oocyte was characterized by being surrounded by follicular cells with a space (antrum) between the cells;
- atretic oocyte was an oocyte containing a pycnotic nucleus and/or a highly irregularly shaped nucleus.

Atretic oocytes were only counted if they could be distinguished from atretic somatic cells [17].

Estimation of the number of oocytes. Estimates of the number of preantral, antral, and atretic oocytes was made on a PC in conjunction with a Nikon E200 microscope (Nikon, Japan) which was fitted with $\times 60$ oil immersion objective with a high numerical aperture (NA: 1.4). Microscopic fields were selected through moving the microscope stage in an equal distance and a systematic uniform random sampling $[13,15]$. A microcator (MT12, Heidenhain, Germany) mounted on the microscope was used in order to measure the movement of the stage in the z-axis. An optical disector design was used to sample the tissue in conjunction with an unbiased sampling frame $[13,15]$. A brief description appears under Figure 2. On average, 250-440 microscopic fields were studied in the animals. Also, 80-120 oocytes were counted and/or analyzed per animal $[13,15,17]$.

Mean oocyte surface area and volume. The mean surface area and volume of the oocytes were measured using a 'number-weighted population mean' method which is called the 'invariator' $[12,14]$. Because the required means were number weighted, the oocytes were sampled with identical probabilities using disectors. A proper design of selection was started with a systematic series of isotropically orientated slices. To sample the oocytes, each slice was subsampled by an optical disector. The target sample was the nucleolus. A slice was subsampled by a sweeping optical plane (optical disector technique) and an unbiased counting frame [17]. The cells whose nucleoli were observed were sampled with identical probabilities, and were used to estimate the size. A brief description is presented under Figure $3[12,14]$.

Statistical methods. $T$-test was used in order to analyze the data. The results are expressed as mean and coefficient of variation $(\mathrm{CV} \%)$, and $\mathrm{p}<0.05$ is considered as statistically significant.

\section{Results}

The histologic section of the ovary in the control and the ND-treated mice is presented in Figure 4. The structural parameters of the normal and the ND-treated animals are presented in Tables 1 and 2. On average, the weight as well as the volume of the ovary, cortex $(\mathrm{p}<0.005)$, and medulla $(\mathrm{p}<0.01)$ decreased by $50 \%$ in ND-treated mice compared to the control group (Table 1). The number of preantral $(\mathrm{p}<0.03)$, antral, and atretic $(\mathrm{p}<0.005)$ oocytes decreased $\sim 54 \%, \sim 87 \%$, and $\sim 91 \%$, respectively in the ND-treated animals $(\mathrm{p}<0.04)$. However, the volume as 


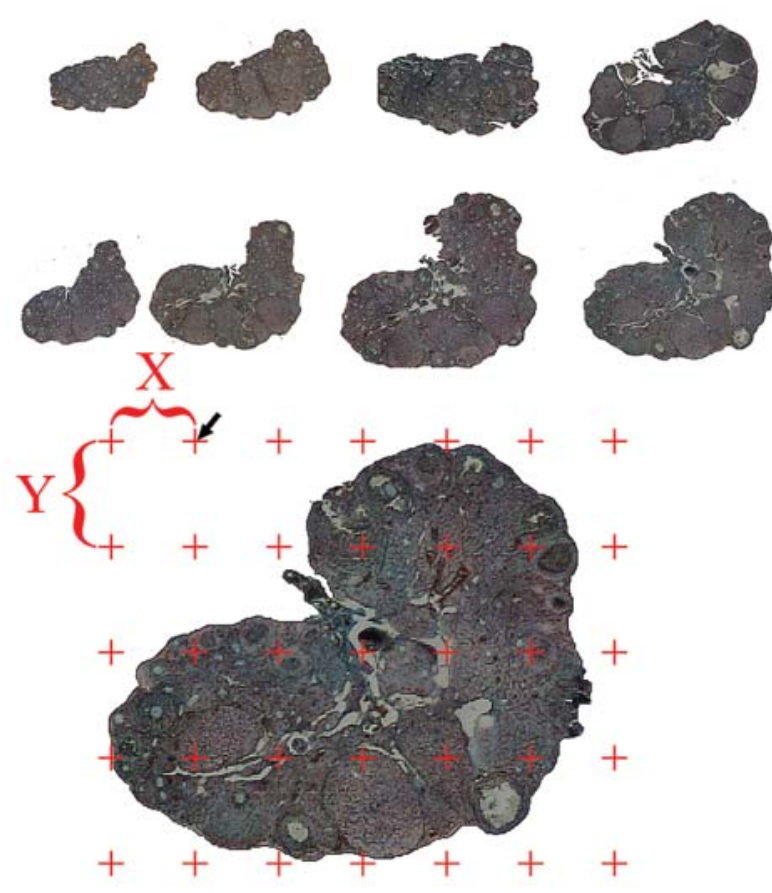

Figure 1. Estimation of the ovary volume using the Cavalieri principle. Eight-twelve sections from each ovary were sampled. A test system of points was overlaid on the image of the tissue using the stereology software designed at our research center. The estimation was made using the following formulas:

$V(\operatorname{Ref}):=A \cdot t$

$A:=a(p) . \Sigma P_{i}$

where " $t$ " is the distance between the sampled sections and the section thickness, " $\Sigma P_{i}$ " is the total number of points hitting the sections of cortex and medulla, and " $a(p)$ " is the area per point which is calculated by multiplying " $\Delta \mathrm{X}$ " by " $\Delta \mathrm{Y}$ ". The arrow indicates the point which is at the right upper corner of the cross

well as the surface area of the oocytes remained unchanged (Plot 1, Table 2).

\section{Discussion}

Estimation of the number of follicles and oocytes has received much attention due to its major role in female reproduction. The appropriate surface area and volume of any cells, including oocytes, are necessary for their normal function. The surface area of oocytes is the entrance of the spermatozoon. Changes in the size of this route may lead to fertilization.

The present investigation has revealed the effects of ND on the histological parameters of the ovary. This study showed that ND induces structural changes, such as a decrease in the number of oocytes. This accords with the study by Cherici Camargo et al. [6], which indicated that the number of antral and atretic follicles was affected by the steroids. They reported
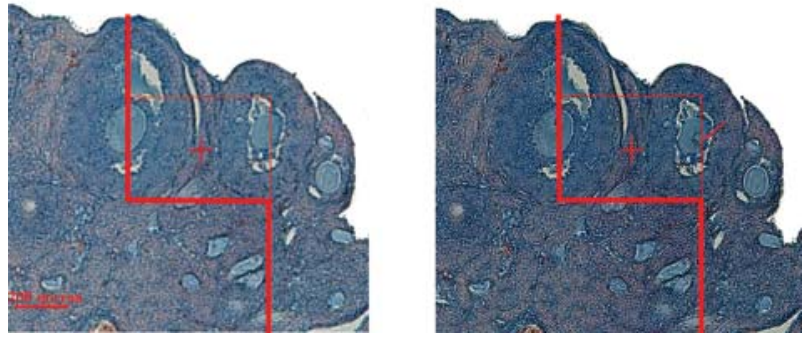

Figure 2. Estimation of the number of oocytes using the optical disector. At each microscopic field, the first $3 \mu \mathrm{m}$ was traversed as a guard area against the cutting artifacts. Then, the nucleoli profiles of the oocytes were counted in the next $10 \mu \mathrm{m}$ of the $15 \mu \mathrm{m}$ section. The nucleoli were counted only if they were inside or partially inside the sampling frame and none of their parts touched the exclusion lines of the frame (the arrow). The numerical density $\left(\mathrm{N}_{\mathrm{v}}\right)$, or number of the cells in the unit volume of the ovary, was estimated using the following formula:

$\mathrm{N}_{\mathrm{v}}:=\frac{\Sigma \mathrm{Q}^{-}}{\mathrm{a}(\text { frame }) \cdot \mathrm{h} \cdot \Sigma \mathrm{P}}$

where , $\Sigma \mathrm{Q}^{-”}$ is the number of the nucleoli sampled in the disector height, , $\Sigma \mathrm{P}$ ” is the total number of the counted frames in all microscopic fields, $a$ (frame) is the area of each frame ( $2342 \mu \mathrm{m}^{2}$ here), and " $h$ " is the height of the disector (10 $\mu \mathrm{m}$ here). The total number of the oocytes was estimated by multiplying the numerical density $\left(N_{\mathrm{v}}\right)$ by the total volume of the ovary

that the weight of ovaries decreased after ND treatment, just the same as the results obtained in the present study. In this study, atrophic changes of the ovary was shown as a quantitative parameter; i.e. the volume. A decrease in the volume of ovary, cortex, and medulla has been reported in our work and this could be due to the reduction in the number of oocytes and follicles. In addition, Gerez et al. [7] reported that rats treated with nandrolone decanoate showed a destruction of the follicular units as well as an absence of corpus luteum in the ovaries. Cherici Camargo et al. [6] reported that the area of corpus luteum decreased in ND-treated animals. Two-dimensional parameters - such as area — can be obtained by measuring the profile section area of the corpus luteum. Area estimation is different from surface area estimation, which should be estimated in 3-D.

Anabolic androgenic steroids induce these effects by dispersing the neuroendocrine function in females $[7,18]$. In the normal female body, a little testosterone is produced, and artificial materials increase the level of anabolic androgens in the blood which will affect the hypothalamic-pituitary-gonadal axis and change the normal functions of the axis. It has been reported that negative feedback is suppressed by high levels of circulating anabolic androgen, thereby reducing the dis- 


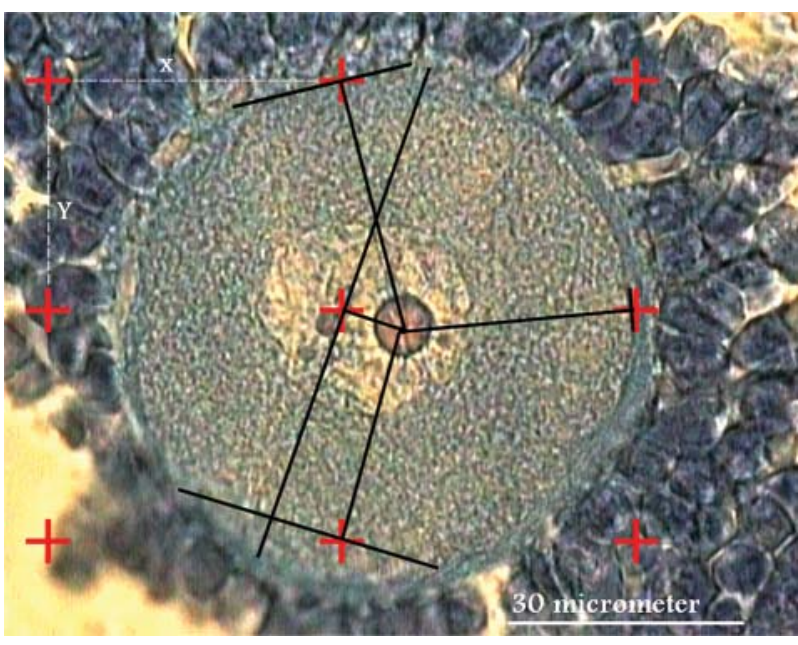

Figure 3. Estimation of the surface area and the volume of oocytes using the invariator. A uniform random quadrangular test system of fundamental area "a" was overlaid on the images, where " $\mathrm{a}=\Delta \mathrm{X} . \Delta \mathrm{Y}$ ". Each sampled nucleolus was adopted as the pivotal point. The vertices were drawn from the pivotal point to all angles of the quadrangular test system which were correlated to the sampled oocyte. Test lines were drawn perpendicular to the vertices at the junction of the vertices and the quadrangles. The surface area and the volume were estimated using the following formulas:

$\overline{\mathrm{S}}:=2 \overline{\mathrm{I}}_{\mathrm{p}}$

$\overline{\mathrm{V}}:=\mathrm{a} \overline{\mathrm{L}}_{\mathrm{p}}$

i.e. the surface area and volume are equal to ' $2 \mathrm{a}$ ' times the mean number of intersections $\left(\overline{\mathrm{I}}_{\mathrm{p}}\right)$ and ' $\mathrm{a}$ ' times the mean total intercept length $\left(\overline{\mathrm{L}}_{\mathrm{p}}\right)$ per pivotal point (hence the subscript ' $p$ '), respectively. Four test lines and eight intersections can be found in the figure

charge of gonadotropin. As a result, an increase in the circulating androgens will inhibit the production and release of $\mathrm{LH}$ and $\mathrm{FSH}$, leading to a decline in the serum levels of LH, FSH, estrogens, and progesterone $[7,18]$. This may result in the inhibition of follicle formation and ovulation. Therefore, the reduction in the above mentioned hormones induces the reduction in the number of the oocytes which had partially grown for ovulation (preantral), those which were growing, and fully matured ones (antral and atretic). However, it did not affect the oocytes' volume and surface area.

In this survey, the invariator (pivotal plane and point) was used to estimate the oocyte's size. There are different methods of local volume estimation including the nucleator and the selector [12-15]. Volume estimation using the nucleator is more common than the pivotal plane and point method described by Cruz-Orive [12, 14]. The advantage of the pivotal plane and point method is the simultaneous estimation of the surface area
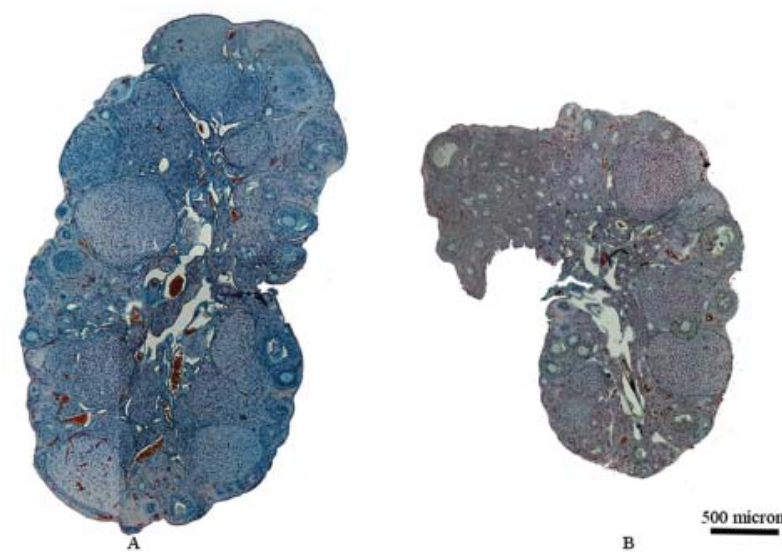

Figure 4. The reconstructed histological section of the ovary in the control (A) and the ND-treated (B) mice. An atrophic change of the ovary is obvious in the ND group

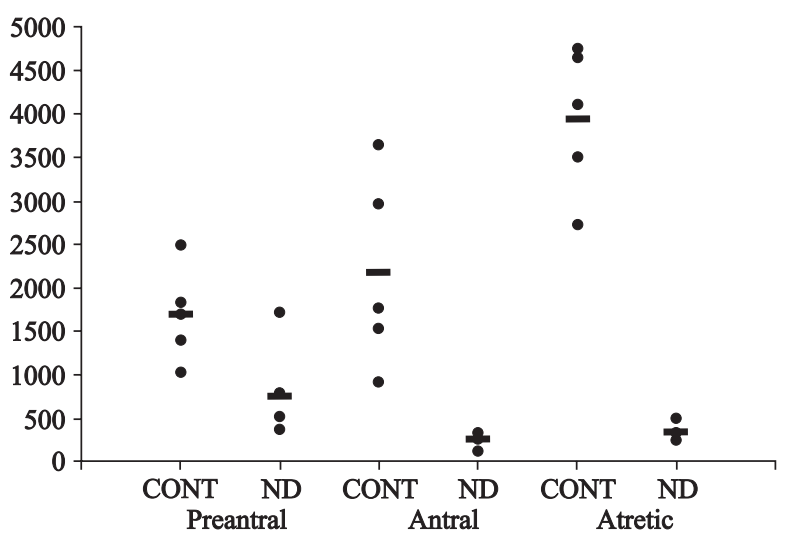

Plot 1. The total number of oocytes in preantral, antral, and atretic follicles of the control (CONT) and the ND-treated (ND) mice

and the volume. The selector is more time-consuming, and not suitable for surface estimation.

It should be noted that shrinkage by paraffin embedding may distort both the volume and the surface area. In order to reduce the shrinkage, embedding of the tissue in resins, such as methyl methacrylate, is recommended.

\section{Conclusion}

Nandrolone decanoate induces a reduction in the number of the oocytes, but not the mean oocyte volume or surface area.

\section{Acknowledgements}

This work was carried out in the Histomorphometry and Stereology Research Center, Shiraz University of Medical Sciences (Shiraz, Iran). The authors ap- 
Table 1. Mean and coefficient of variation $(\mathrm{CV} \%)$ of the body weight $(\mathrm{g})$, ovary weight $(\mathrm{mg})$, and volume $\left(\mathrm{mm}^{3}\right) \mathrm{of} \mathrm{the}$ ovary, cortex, and medulla of the ovary, among control and experimental animals $(\mathrm{n}=5)$

\begin{tabular}{|l|c|c|c|c|c|}
\hline Groups & Body weight & Ovary weight & Ovary volume & Cortex volume & Medulla volume \\
\hline Control & $31.8(0.04)$ & $10.4(0.90)$ & $5.0(0.30)$ & $4.8(0.29)$ & $0.2(0.50)$ \\
\hline Experimental & $31.9(0.04)$ & $5.2(0.10)^{*}$ & $2.2(0.30)^{*}$ & $2.1(0.28)^{*}$ & $0.1(0.5)^{* *}$ \\
\hline
\end{tabular}

${ }^{*} \mathrm{p}<0.005$, experimental vs. control group; ${ }^{* *} \mathrm{p}<0.01$, experimental vs. control group

Table 2. Mean and coefficient of variation $(\mathrm{CV} \%)$ the total number of oocytes, the mean volume $\left(\mu \mathrm{m}^{3}\right)$, and the mean surface $\left(\mu \mathrm{m}^{2}\right)$ of the oocytes in the ovary among control and experimental animals $(\mathrm{n}=5)$

\begin{tabular}{|l|c|c|c|c|c|c|c|c|c|c|}
\hline Groups & \multicolumn{3}{|c|}{ Number } & \multicolumn{3}{c|}{ Volume } & \multicolumn{3}{c|}{ Surface } \\
\hline & Preantral & Antral & Atretic & Preantral & Antral & Atretic & Preantral & Antral & Atretic \\
\hline Control & 1,690 & 2,100 & 3,900 & 86,000 & 110,000 & 27,000 & 9,000 & 9,900 & 4,700 \\
& $(0.29)$ & $(0.52)$ & $(0.2)$ & $(0.27)$ & $(0.48)$ & $(0.33)$ & $(0.24)$ & $(0.28)$ & $(0.21)$ \\
\hline Experimental & 760 & 260 & 340 & 70,000 & 85,000 & 34,000 & 8,000 & 9,900 & 4,000 \\
& $(0.73)^{*}$ & $(0.30)^{* *}$ & $(0.26)^{* *}$ & $(0.37)$ & $(0.60)$ & $(0.47)$ & $(0.27)$ & $(0.42)$ & $(0.47)$ \\
\hline
\end{tabular}

${ }^{*} \mathrm{p}<0.03$, experimental vs. control group; ${ }^{* *} \mathrm{p}<0.005$, experimental vs. control group

preciate Dr Ali Musavi and Mr Hashem Zare as the stereology software developers. Miss Elham Nadimi, Mr Mehrdad Azadi and Ms Zahra Keshavarz are acknowledged for their technical help. The Research Improvement Center of Shiraz University of Medical Sciences and Ms Afsaneh Keivanshekouh are also appreciated for improving the use of English in this manuscript.

\section{Conflict of interest}

The authors report no conflicts of interest.

\section{References}

1. Blasberg ME, Langan CJ, Clark AS. The effects of alphathyltestosterone,methandrostenolone and nandrolone decanoate on the rat estrous cycle. Physiol Behav. 1997;61:265-272 .

2. Iriart JAB, Andrade TM. Body-building, steroid use, and risk perception among young body-builders from a low-income neighborhood in the city of Salvador, Bahia State, Brazil. Toxicol Appl Pharmacol. 2002;18:71-81.

3. Yu-Yahiro J, Michael RH, Nasralah DV, Schofield B. Morphologic and histologic abnormalities in female and male rats treated with anabolic steroids. Am J Sports Med. 1989;17:686-689 .

4. Noorafshan A, Karbalay-Doust S, Ardekani FM. High doses of nandrolone decanoate reduce volume of testis and length of seminiferous tubules in rats. APMIS. 2005;113:122-125.

5. Karbalay-Doust S, Noorafshan A. Stereological study of the effects of nandrolone decanoate on the rat prostate. Micron. 2006;37:617-623.

6. Cherici Camargo IC, Barreiros de Souza R, de Fátima Paccola Mesquita S, Chuffa LG, Frei F. Ovarian histology and follicular score in female rats treated with nandrolone decanoate and submitted to physical effort. Acta Biol Hung. 2009;60:253-261.
7. Gerez JR, Frei F, Camargo IC. Histological assessment of ovaries and uterus of rats subjected to nandrolone decanoate treatment. Contraception. 2005;72:77-80.

8. Karbalay-Doust S, Noorafshan A, Ardekani FM, Mirkhani H. The reversibility of sperm quality after discontinuing nandrolone decanoate in adult male rats. Asian J Androl. 2007;9:235-239.

9. Bronson FH, Nguyen KQ, Delarosa J. Effect of anabolic steroid on physiological characteristics of female mice. Physiol Behav. 1996;59:49-55.

10. Strauss RH, Liggett MT, Lanese RR. Anabolic steroid use and perceived effects in ten weight-trained women athletes. JAMA. 1985;253:2871-2873.

11. Howe GR, Morello CJ. Effects of an anabolic steroid on reproduction in female rats. Steroid. 1985;45:495-501.

12. Cruz-Orive LM, Ramos-Herrera ML, Artacho-Pérula E. Stereology of isolated objects with the invariator. J Microsc. 2010;240:94-110.

13. Mühlfeld C, Nyengaard JR, Mayhew TM. A review of stateof-the-art stereology for better quantitative 3D morphology in cardiac research. Cardiovasc Pathol. 2010;19:65-82.

14. Cruz-Orive LM. A new stereological principle for test lines in three-dimensional space. J Microsc. 2005;219(Pt 1):18-28.

15. Hyde DM, Tyler NK, Plopper CG. Morphometry of the respiratory tract: avoiding the sampling, size, orientation, and reference traps. Toxicol Pathol. 2007;35:41-48.

16. Nyengaard JR, Gundersen HJG. The isector: a simple and direct method for generating isotropic, uniform random sections from small specimens. J Microscopy. 1992;165;427$-431$.

17. Myers M, Britt KL, Wreford NG, Ebling FJ, Kerr JB. Methods for quantifying follicular numbers within the mouse ovary. Reproduction. 2004;127:569-580.

18. Bonetti A, Tirelli F, Catapano A et al. Side effects of anabolic androgenic steroids abuse. Int J Sports Med. 2008;29: 679-687.

Submitted: 28 August, 2011 Accepted after reviews: 8 January, 2012 